\title{
Guarded Process Spaces (GPS): A Navigation System towards Creation and Dynamic Change of Healthcare Processes from the End-user's Perspective
}

\author{
Claudia Reuter ${ }^{1}$, Peter Dadam², Stephan Rudolph ${ }^{3}$, Wolfgang Deiters ${ }^{3}$, \\ Simon Trillsch ${ }^{4}$ \\ ${ }^{1}$ Zühlke Management Consultants AG, Wiesenstr. 10a, \\ 8952 Schlieren, Switzerland \\ claudia.reuter@zuehlke.com \\ ${ }^{2}$ Ulm University, Albert-Einstein-Allee 11, 89081 Ulm, Germany \\ peter.dadam@uni-ulm.de \\ ${ }^{3}$ Fraunhofer Institute for Software and Systems Engineering, Emil-Figge-Str. 91, \\ 44227 Dortmund, Germany \\ \{stephan.rudolph, wolfgang.deiters\}@isst.fraunhofer.de \\ ${ }^{4}$ University Hospital of Giessen and Marburg, Baldingerstr., 35033 Marburg, Germany \\ trillsch@students.uni-marburg.de
}

\begin{abstract}
Efficient process management becomes increasingly crucial for hospitals to survive on a competitive market. Process management in this domain must comply with individual conditions of patients and quickly react to changing requirements and organizational parameters. With Guarded Process Spaces (GPS) we developed a formally based concept that makes it possible to enable end-users to create and flexibly change processes themselves. Our approach makes use of existing BPM technology while abstracting from technical interfaces and system-specific modeling paradigms. In this way, it provides the basis to gain user acceptance and to achieve technological independence.
\end{abstract}

Keywords: Healthcare process, clinical pathway, process flexibility, domain specific languages

\section{Motivation}

Today, healthcare providers are facing the challenge of delivering high-quality services while coping with increasing costs due to demographic change and medical progress. In response to this, hospitals start with the introduction and deployment of standard processes (so called "clinical pathways") to organize the treatment of patients according to a common set of symptoms, a diagnosis, or a therapy. In principle, modern BPMSs (Business Process Management Systems) could help to support clinical pathways in practice and to reduce administrative workload by instantiating pathway templates for patients, documenting and monitoring their 
progress, and managing work lists for doctors and nurses. However, in spite of their potential benefit, BPMSs are barely in use in healthcare environments until now.

Originally, BPMSs have been developed to support production processes in industry. In such settings processes typically shall be executed exactly as preplanned to ensure that the goals in terms of quality and cost are met for all the products. In clinical pathways, however, the focus must be put on the patient as individual being. Therefore, in order to support clinical pathways using BPMSs one must be able to solve the conflict between standardization of treatment processes on the one hand and flexible deviation from standards due to case-based considerations on the other hand.

The fact that healthcare processes pose challenges to traditional BPMSs is not new to the scientific community; frequently, they even serve as motivation for researchers to investigate new approaches [1, 2]. In fact, flexibility and adaptivity during process execution are broadly addressed in BPMS related research in the meanwhile [3]. Therefore, in the near future, we can expect that BPMSs come onto the market, which allow for more process flexibility at runtime. However, supporting flexibility at the BPM system level and making this feature usable by end-users are two different stories. A direct interaction with a BPMS, e. g., to insert or to postpone a task requires profound technical skills, which the medical staff is not able and not willing to acquire. This means, one must find a solution, which enables end-users to flexibly adjust clinical pathways according to the individual demands of a patient, but does not force them to acquire deep system near skills in order to perform this task.

In context of the SPOT project (Service-based and Process-oriented Orchestration Technology $)^{1}$ we developed a concept and a prototype demonstrating that such kind of system can be realized. The concept is based on the notion of "Guarded Process Spaces" (GPS). The analogy to GPS navigation devices is intentional, because like such devices, which can answer the question "Which roads are available to me now?", Guarded Process Spaces provide maps of possible directions a process can take and guide the user's decision making as to which paths they can follow to reach a valid goal.

In this paper, we will focus on the benefit of GPS from the end-user's perspective. After a discussion of related work in chapter 2, we will introduce a novel navigation paradigm towards process modeling, which is realized based on GPS in section 3. In chapter 4 we will explain the technical implementation of GPS by way of a practical example from the healthcare domain. After that, we will discuss requirements on BPMSs in terms of process flexibility and show how these demands can be fulfilled by GPS in combination with existing approaches from chapter 2 . Finally, we will give a short summary and an outlook on our future work.

\section{Related Work}

Process modeling languages used by process experts are usually too complicated for end-users to model processes themselves. Therefore, domain specific languages (DSL) are developed to facilitate process modeling for end-users in their application

\footnotetext{
${ }^{1}$ See http://www.spot.fraunhofer.de
} 
domain, like, e. g., public administration [4], workflow-based web applications [5], integrated care [6], or medical guidelines [7]. Another example is the feature modeling approach for modeling variability in product families [8], which has already been applied to process management as well [9]. However, the provision of DSLs alone is not sufficient without ensuring that the technical process templates which are derived from such DSLs can be correctly executed by a BPMS. This means, that DSLs without a proper and suitable formal basis are not very helpful to achieve this goal. Due to the lack of formality of DSLs, they are often transformed into formal languages, like e. g. Petri Nets [10], to perform correctness checks. However, this doesn't prevent the creation of erroneous process templates, which have to be corrected afterwards which, in turn, delays the whole development process, decreases user acceptance, and is certainly not acceptable in case of ad-hoc changes. Instead, one has to provide a modeling environment which guides the user in such a way that "technical” modeling errors (like deadlocks, incorrect or incomplete data flows, etc.) are excluded as far as possible; and the same must hold for ad-hoc deviations at runtime. The "correctness by construction" approach developed in the ADEPT project [11-12] proved to be the best suited one for that purpose and, therefore, was very influential for the development of respective concepts in GPS.

Assumed, we have a DSL with an adequate formal basis, the question remains how to offer the required flexibility to an end-user (e.g., a physician) such that she herself is able to adjust a clinical pathway according to the individual demands of her patient.

In recent years, the scientific community has made great technical advances especially with regard to dynamic process management. [13], e.g., deals with shifting existing tasks within a process instance under correctness constraints. Other authors suggest maintaining the standard way of proceeding together with its variations within the same process template [14-16]. Using respective workflow patterns, placeholder activities (like, e.g., Proclets [17]) or variation points, it is possible to indicate the positions, where alternative routes may be chosen or even created at runtime. The advantage of these approaches is that end-users don't have to comprehend the process template and the usage of change operations to deviate from the standard proceeding. The disadvantage is that such approaches require that the positions where alternative routes may be chosen have to be fixed in advance, which does not reflect the reality in healthcare. E. g., certain conditions, such as infections, can occur at any point in time. Therefore, these simple solutions are falling short of covering these demands. Endusers must be able to flexibly change the process structure at runtime.

In [11-12] it is illustrated how an end-user interface to perform an ad-hoc change could be implemented using the application programming interface of the ADEPT2 system. In this example the user wants to insert a new task into the process. After having selected the desired task, the system shows him a simplified process graph within which he can select a process step. This selection informs the system that the new task shall become executable after this task. Then the system allows him to choose another process step, before which the new task must be completed. Based on this information the system determines where and how the new task is inserted (as serial or parallel step) and performs the necessary transformations of the process graph. Although this approach does not require system-near knowledge to perform such a task, it confronts the user with a different and rather "technical view" of the clinical pathway compared to the GPS approach for process modeling. The goal of 
GPS is to apply the same user-oriented metaphors for ad-hoc deviations as in case of process modeling.

\section{Guarded Process Spaces: Applying the Navigation Paradigm to Process Modeling}

Our investigations during the SPOT project were driven by the following objectives: Firstly, it must be possible for end-users to design process templates from their business point of view and to automatically execute them using a chosen BPM system. Secondly, end-users must be enabled to change processes both at modeling time and at runtime on a case by case basis.

As already mentioned, we apply a navigation paradigm to process modeling which we call Guarded Process Spaces (GPS). Due to lack of space, we cannot describe this approach in detail in this paper. Instead, we will introduce GPS informally and point out their usage by the clinical staff. A detailed and formal description can be found in $[18,19]$ (where GPS correspond to so called "SPF-type graphs"). From a user's point of view a GPS acts like a navigation system, which uses a given set of streets to offer routes and alternative routes from the current location to the desired destination. At technical level a GPS consists of a set of nodes and has a tree-like structure. The nodes represent navigation points, which are used to implement rules on the selection of process activities. The root of the GPS represents the starting point, from where "travels" can be planned. Like a navigation system, the Guarded Process Space indicates all potential traveling options. By selecting an option, a user is travelling to the next navigation point within the coordinate network; there, further traveling options are available. With every step that a user takes, the amount of traveling options to approach the final destination decreases. The selected route from the starting point to the destination defines an executable clinical pathway.

We illustrate process modeling from the user's perspective by means of a practical example. During their inpatient stay, some patients experience shortness of breath, the reason for which can be a bacterial pneumonia or a left heart failure. The clinical staff shall develop clinical pathways that coordinate the diagnostics of one of these diseases as well as both of them together. Fig. 1 illustrates how this is done. According to the GPS, the pathway for all clinical diagnostics is divided into different categories, such as "Radiology" or "Laboratory". These categories represent the navigation points within the GPS and may contain further specialized navigation points. E. g., the navigation point "Radiology" comprises a chest X-ray examination and encapsulates further radiological activities within navigation point "Additional radiology". Now, the modeler team can decide whether or not additional examinations should be scheduled within the clinical pathway by default.

The pathway modeler team navigates through the GPS in order to develop a clinical pathway for the diagnostics of bacterial pneumonia. All tasks they select will be included in the pathway. After having made all these choices, the clinical pathway is determined and an executable process template can be generated. As we will see 
later, this does not mean that the resulting clinical pathway is now completely inflexible.

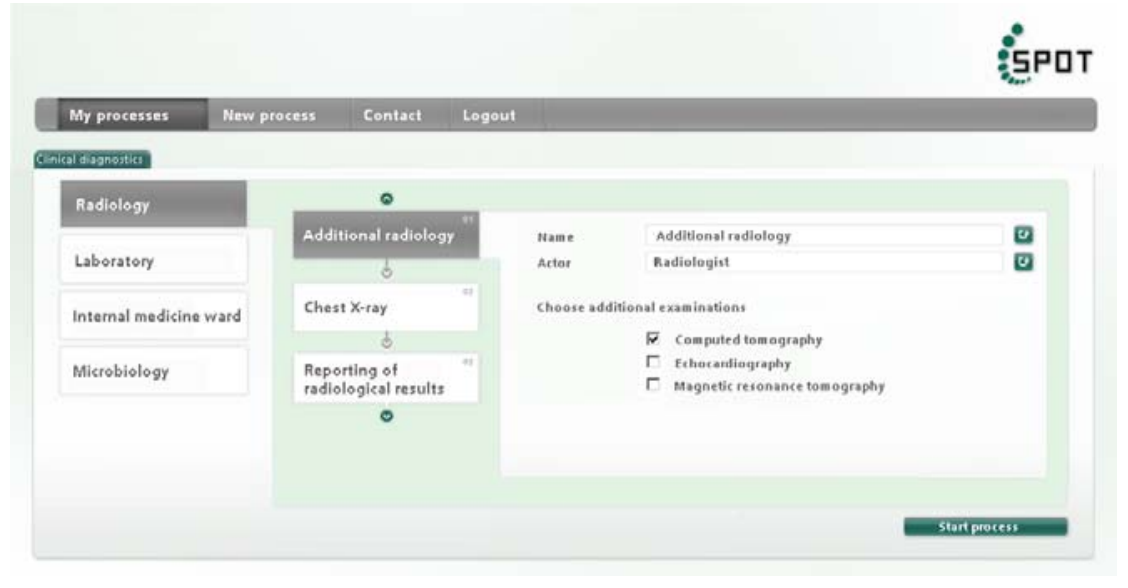

Fig. 1. Modeling clinical pathways from the end-users' perspective.

If needed, the end-user (e.g., the ward physician) can repeat tasks or can "reactivate" deselected tasks in the context of ad-hoc deviations at runtime to adjust the clinical pathway to the individual needs of a specific patient.) By performing process modeling this way, the end-users can mentally completely stay in their "world" and just select the tasks to be performed. All the other aspects like setting up the resulting control flow, the data flow, deadlock avoidance, and other things are handled at GPS system level and do not bother him. In addition, the GPS also "knows" which tasks depend on each other or, just the opposite, exclude each other. This means that certain kinds of mistakes are automatically avoided.

\section{Implementation of Guarded Process Spaces}

Fig. 2 illustrates how the support for this navigation and decision process is implemented at the technical level. The graph on the left side corresponds to the GPS and the graph on the right side represents the currently developed clinical pathway. The nodes in the GPS graph represent either tasks or logical operators like, e.g., AND, XOR, OR, and OPT (for optional). In Fig. 2, the root node "Clinical diagnostics" is connected with an AND-operator, i. e., all child nodes have to be selected. According to the OPT-operator at "Additional radiology", it is possible to choose an arbitrary number of child nodes or none at all.

Since AND-operators don't leave many options, a big part of the clinical pathway can be automatically derived from the GPS in this case. E. g., a chest X-ray always includes some laboratory examinations and reporting to the ward physician. With regard to the possible variations "Additional radiology" and "Additional laboratory" the modeler have to decide which ones (if any) shall occur in the clinical pathway. 


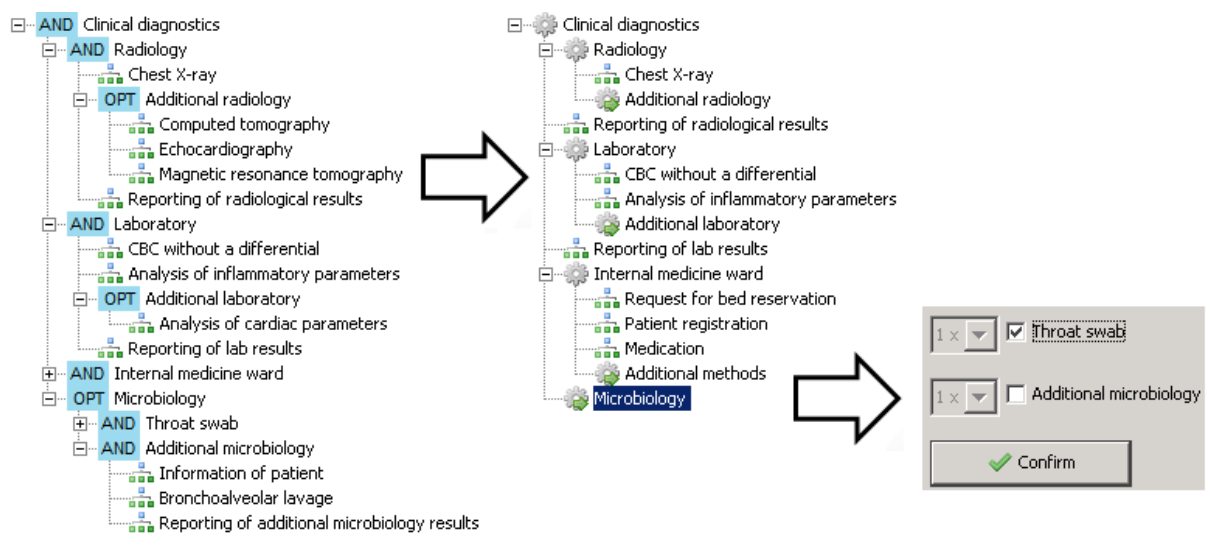

Fig. 2. Modeling of a clinical pathway for diagnostics of bacterial pneumonia based on GPS.

For diagnostics of pneumonia a throat swab is required. Therefore, the modeler team selects "Throat swab" from the navigation point "Microbiology". After that, the clinical pathway for bacterial pneumonia is complete. In order to obtain the pathway for diagnostics of left heart failure, the modelers must only take a slightly different route with respect to some navigation points.

As indicated above, the GPS can also contain constraints determining the execution order of process activities within the clinical pathway. Constraints are defined as edges connecting GPS nodes on a horizontal axis. E. g., it can be expressed, that the activity "Reporting of radiological results" must not be scheduled as long as "Chest X-ray" and optionally "Additional radiology" are not finished. One could also state that some nodes may require or exclude other nodes. In addition, one can specify constraints for node cardinalities. A node cardinality defines the maximum number of times that a clinical pathway may contain a set of process activities. This feature can be used to model cyclic treatments. In $[18,19]$ formal correctness criteria are defined to ensure that constraints cannot contradict each other and are in conformance with the GPS structure and its logical operators. Due to flexibility options, kind and amount of data objects the system has to deal with at runtime may vary. This means, the application components that implement process activities must cope with variable data input and output. In [19], an interface specification of process activities is defined, which among others specifies both mandatory and optional data input and output. Moreover, it is described how data dependencies can be considered at GPS level. Further aspects, like e. g. data storage and versioning, must be handled at technical level and are out of scope of the GPS approach.

In general, the more process knowledge a GPS captures, the easier the creation of clinical pathways and - finally - executable process templates becomes for the clinical staff. To make the creation of clinical pathways by end-users as easy as described above, the GPS graph must comprise all potential tasks, choices, and relevant constraints of the considered application area. The range of applicability, the acceptance of the GPS based modeling environment, and the resulting executable clinical pathways depend on the degree to which this graph covers the application area. The clinical staff can (and must) help to develop the initial hierarchical structure 
of a GPS and to identify relevant process activities. The implementation of the GPS itself, the implementation of activities by executable application components, user interfaces, and task-specific control and data flow aspects will require IT-specialists. To decrease the complexity of this task one can take a stepwise refinement approach by first modeling the GPS graph rather coarsely and re-examine it with end-users using the modeling environment. Then one refines one or several nodes and checks it again, etc.

\section{Enabling Process Change from the End-user's Perspective}

With GPS, end-users are now in the position to create various clinical pathways using the offered navigation paradigm. However, in healthcare it is often not possible to plan the complete treatment process in advance. Instead, the treatment process develops depending on further insights gained during the execution of the process. Under certain circumstances, it may even become necessary to abandon the original plan, to return to a specific point, and to choose a different option. Therefore, in this chapter we describe healthcare-specific requirements on process flexibility and discuss how they can be addressed using GPS in combination with existing BPMSs.

Flexible extension of pathway instances. Although, the modelers of clinical pathways determine medical treatment to a large extent, some decisions can only be made by the doctor in charge of the patient. With regard to our pathway example, each patient who is suspected to suffer from bacterial pneumonia will undergo a throat swab. After that, the further proceeding depends on whether the finding is positive or negative. Assuming, the finding is positive, then the GPS specifies the available options; i. e., to carry out a bronchoalveolar lavage (cf. Fig. 2). When selecting this choice, the GPS may enforce an application constraint, e.g., that a bronchoalveolar lavage is only possible if the patient has been transferred to the isolation ward first in order to prevent the spreading of the disease. If this case is not rare to occur, the pathway modelers will have anticipated this additional examination along with the associated tasks and may offer it in terms of conditional branches, placeholder activities or variation points.

Minor deviations from clinical pathways. Clinical pathways specify the standard way of proceeding. Accordingly, the pathway for diagnostics of bacterial pneumonia only schedules a chest X-ray in the course of radiological examinations. However, in certain cases, it can become necessary to perform additional procedures. For example, the ward physician examines the chest X-ray and the lab results of her patient. As it is not possible to confirm the diagnosis on the basis of these findings, she decides on scheduling a computed tomography. This examination is not part of the clinical pathway by default. Therefore, in the context of radiology a deviation from the pathway occurs. After changing the configuration of the navigation point "Additional radiology”, the new task is added to the work list of the responsible radiologist. Fig. 3 indicates how end-users can handle minor deviations from clinical pathways using the GPS approach. 


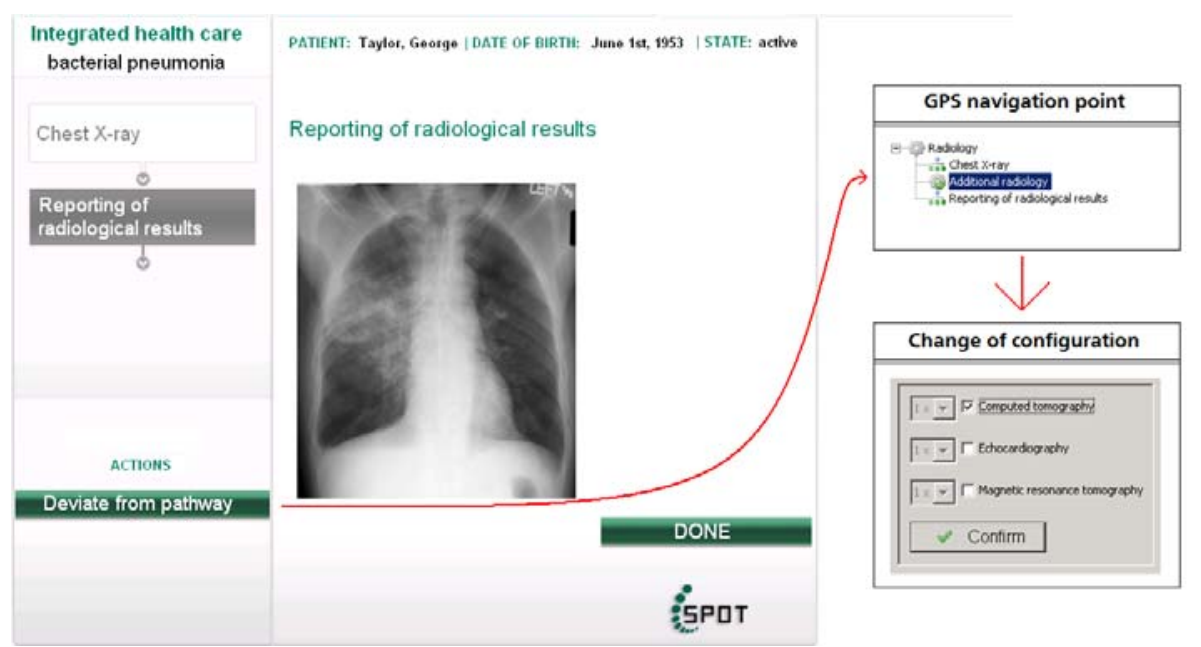

Fig. 3. Ad-hoc change of pathway instances from the end-user's perspective.

First, one has to signal the need for deviation from the process standard by clicking the button "deviate from pathway" within the user interface. Then, the end-user can choose additional radiological examinations, which are available in the context of the navigation node "Additional radiology" of the GPS. By selecting one or more examinations, the end-user changes the pathway schedule in an ad-hoc manner. Provided that the position where such a deviation may happen is known in advance, it is sufficient to use conditional branches, placeholder activities, or variation points in order to technically realize this ad-hoc change. Frequently, it is not so clear at which point of time a variance arises, however. Regarding the GPS, it is not important when the physician decides to deviate from the pathway. The GPS may define constraints that determine the position where a computed tomography should be performed at best. However, if the execution flow has already passed this position, the examination may be scheduled at the next possible place.

Complex deviations from clinical pathways. The detection of second diagnoses or complications may result in more complex deviations from clinical pathways. E. g., a physician chose the pathway for diagnostics of bacterial pneumonia for her patient. During the treatment she discovers symptoms that indicate a left heart failure. She decides to modify the process in a way that it also covers the procedures for this second diagnosis. So, changes can not only occur at isolated spots, but may affect several regions of a process. In our example the doctor would have to insert the activities "Analysis of cardiac parameters" within "Laboratory" and "Echocardiography" within "Additional radiology". Such complex deviations may significantly increase the effort of physicians to perform the change of the pathway and thus raise the probability of errors. Therefore, if the pathway modelers have anticipated such a situation, they could have provided process variants, which encapsulate all the changes that have to be made to perform a pathway modification 
of this kind. In this way, it is even feasible to specify standard ways of proceedings in case of deviations from clinical pathways [19].

Since process variants automate the execution of change operations, it must be ensured that they are in conformance with the medical treatment and the procedures, which have been undertaken so far. As a GPS already determines all the routes that processes may take, possible variations, and relations between certain activities, it can also be used to verify that changes in the context of a process variant do not contradict previous treatment.

\section{From GPS-based Clinical Pathways to Executable Processes}

The clinical pathway conforms to the tree-like structure of the GPS graph, but contains only those process activities, which have been selected for the pathway under consideration (cf. Fig. 2). To obtain independence from a specific BPMS, the GPS graph as well as this "clinical pathway graph” serve as a neutral representation which is mapped to process templates of the chosen BPMS to achieve executable processes. To support the full spectrum of possibilities as well as to make this mapping simple, the ideal target BPMS should support the full spectrum of ad-hoc deviations as provided by ADEPT2 [1, 11-12], for example, late binding of dynamically composed complex activities like those described in [15] or as provided by YAWL's proclet approach [17], as well as process variants like those described in [16].

Among these desirable BPMS features, the requirement for the full spectrum of supported ad-hoc deviations is the most relevant one. If this feature is present, concepts like late binding and process variants can be handled by the mapping layer which acts as a broker between the GPS runtime for the "clinical pathway graph" and the underlying BPMS. If this feature is missing or available only in a rather limited fashion then the mapping will result in complex process graphs, because now the most relevant choices and variants have to be incorporated in the process graph from the very beginning. Besides complexity aspects, incorporation of all choices and variants does not only contradict the concept of clinical pathways that must define standards of medical treatment instead of all possible variations; it also cannot provide the full spectrum of flexibility, which is needed in the clinical domain, as we will show in fig. 4.

Among the available BPMS ADEPT2 [1, 11-12] resp. its commercial version, the AristaFlow $^{\circledR}$ BPM Suite ${ }^{2}$ was closest to the "ideal" BPMS sketched above. It, therefore, was selected as the target BPMS for the proof of concept prototype. In [19] one can find the formally defined transformation rules according to which the compilation to GPS-based clinical pathways to the ADEPT2 process model takes place. Fig. 4 shows in which way the process activities within the context of "Radiology" (cf. Fig. 2) can be mapped to executable ADEPT2 process templates. As radiology comprises both default and optional activities, there are two mapping alternatives in ADEPT2: Either conditional branches are used or the additional activities can be inserted into the process instance on demand.

\footnotetext{
${ }^{2}$ See http://www.AristaFlow.com
} 

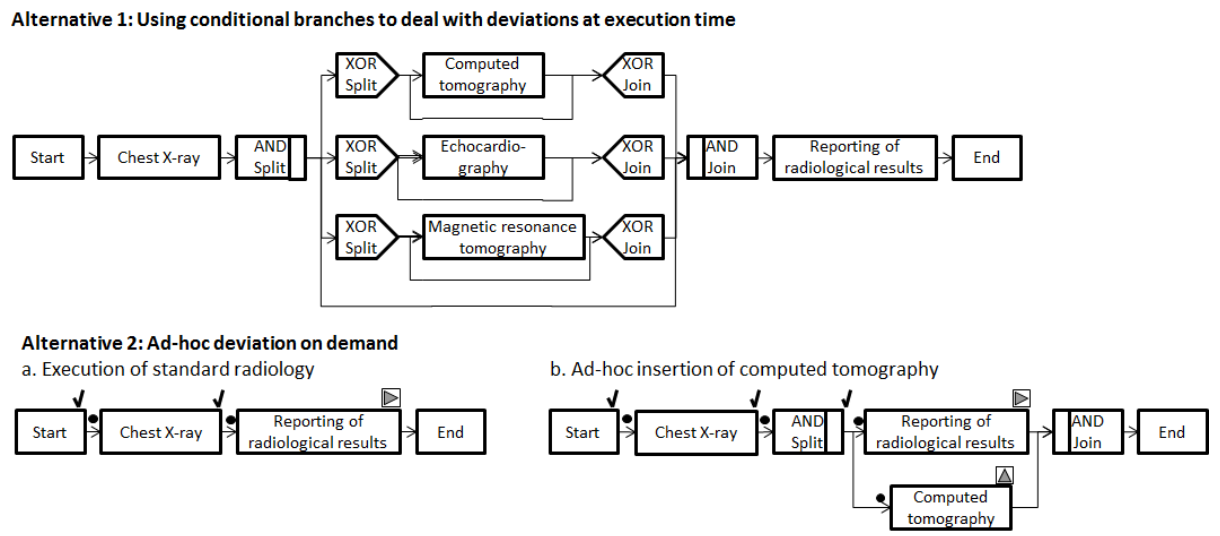

Fig. 4. Mapping the "clinical pathway graph” to ADEPT2 process templates.

According to the constraints of the GPS, the reporting activity should follow the examinations, whereby the chest X-ray has to be performed first. As several or none of the additional examinations can be selected, in alternative 1 we have to insert a complex construct consisting of a parallel as well as three conditional branches. In alternative 2, we only schedule the default activities and perform ad-hoc insertions on demand depending on the current state of the execution. In Fig. 4.a, the reporting activity has already started as a deviation occurs. Thus, it is not possible to insert the computed tomography directly after the chest X-ray, anymore. Instead, it can be added in parallel to the running activity as illustrated in Fig. 4.b. This example shows that the mapping between pathway graph and process template is much simpler and more flexibly than realizing complex workflow patterns. Furthermore, one can clearly distinguish the process standard from its variations. Consequently, BPMSs providing comprehensive support for ad-hoc changes at the API level like, e.g., ADEPT2, are ideal candidates for this approach. As indicated above, other BPMSs can be supported as well, but one is faced with limited flexibility and with more complex implementations of the mapping and runtime layer to compensate the missing functionality.

\section{Summary}

In spite of their potential benefits, BPMSs are not broadly used in healthcare settings yet. In order to be accepted by end-users, the technology has to fulfill the following requirements: Clinical staff must be enabled to model executable process templates by themselves. Moreover, end-users must have the possibility to flexibly adapt running process instances according to the individual demands of their patient. With Guarded Process Spaces (GPS), we presented an approach which uses a "navigation paradigm" to guide end-users in modeling clinical pathways as well as to assist them to perform ad-hoc deviations at runtime for a patient with specific needs. We showed how this approach supports users to select the necessary tasks in the right order and how tasks 
can be automatically inserted when required in the given context. We also gave some insights how this guidance is reflected in the underlying implementation. The approach is based on a sound formal concept which could only be sketched here due to lack of space, however. A comprehensive description can be found in [19]. Another goal of the SPOT project was to base creation and change of clinical pathways on a system-neutral, conceptional layer, which is independent from specific process modeling languages and BPMSs. We provide mapping functions to transform clinical pathways derived from GPS into executable process templates of the chosen target engine and verified this approach by a proof of concept implementation using ADEPT2. In this way, the user interfaces for process modeling and adaptation can remain the same, even if the underlying BPM-technology changes.

In the context of the current project "eBusiness Platform for Healthcare”, we are planning to use the GPS approach to develop medical processes crossing the border of individual healthcare provider institutions. By doing so, GPS are leveraging execution of integrated workflows based on collective knowledge and in spite of heterogeneous system environments.

\section{Acknowledgements}

We want to thank the AristaFlow team, especially Kevin Göser, for the support during the development of the proof of concept prototype.

\section{References}

1. Reichert, M., Dadam, P.: $\mathrm{ADEPT}_{\text {flex }}$ - Supporting Dynamic Changes of Workflows Without Losing Control. In: Journal of Intelligent Information Systems 10, pp. 93-128 (1998)

2. Dadam, P., Reichert, M., Kuhn, K.: Clinical Workflows - The Killer Application for Process-oriented Information Systems?. Proc. Int'l Conf. on Business Information Systems, BIS 2000, 4th Int'l Conf., Poznan, Poland, April 2000, Springer-Verlag, pp. 36-59 (2000)

3. Weber, B., Sadiq, S., Reichert, M.: Beyond Rigidity - Dynamic Process Lifecycle Support A Survey on Dynamic Changes in Process-aware Information Systems. Computer Science Research \& Development, Vol. 23, No. 2, 2009, special issue on "Flexible Process-aware Information Systems", pp. 47-66 (2009)

4. Becker, J., Pfeiffer, D., Räckers, M.: Domain Specific Process Modelling in Public Administrations - The PICTURE-Approach. In: Electronic Government, Lecture Notes in Computer Science 4656, pp. 68-79 (2007)

5. Freudenstein, P., Buck, J., Nussbaumer, M., Gaedke, M.: Model-driven Construction of Workflow-based Web Applications with Domain-specific Languages. Proc. of the $3^{\text {rd }}$ Int'l Workshop on Model-driven Web Engineering (MDWE’07), Como, Italy, 2007

6. Neuhaus, J., Houta, S., Reuter, C.: Ansätze bei der Umsetzung von Behandlungsplanpfaden - Flexibilisierungskonzepte am Beispiel der Behandlung von Wirbelsäulenerkrankungen. In: Hellmann, W., Eble, S. (eds): Ambulante und Sektoren übergreifende Behandlungspfade, pp. 79-97, Medizinisch Wissenschaftliche Verlagsgesellschaft Berlin (2010)

\footnotetext{
${ }^{3}$ See http://www.ebpg-nrw.de/
} 
7. De Clercq, P., Kaiser, K., Hasman, A. : Computer-interpretable Guideline Formalisms. Ten Teije, A. et al. (eds.): Computer-based Medical Guidelines and Protocols: A Primer and Current Trends, IOS Press, pp. 22-43, 2008

8. Kant, K., Cohen, S., Hess, J., Nowak, W., Peterson, S.: Feature-oriented domain analysis (FODA) feasibility study. Technical report CMU/SEI-90-TR-21, Software Engineering Institute, Carnegie Mellon University, Pittsburgh, USA, 1990

9. Puhlmann, F., Schnieders, A., Weiland, J., Weske, M.: Variability Mechniasms for Process Models. PESOA-Report TR 17/2005, Process Family Engineering in Service-Oriented Applications (PESOA), 2005

10. Beccuti, M., Bottrighi, A., Franceschinis, G., Montani, S., Terenziani, P.: Modeling Clinical Guidelines through Petri Nets. Combi, C., Shahar, Y., Abu-Hanna, A. (eds.): Proc. of the $12^{\text {th }}$ Conf. on Artificial Intelligence in Medicine (AIME’09), pp. 61-70, SpringerVerlag Berlin, Heidelberg, 2009

11. Dadam, P., Reichert, M.: The ADEPT Project: A Decade of Research and Development for Robust and Flexible Process Support. Computer Science - Research \& Development, Vol. 23, No. 2, special issue on "Flexible Process-aware Information Systems", pp. 81-98 (2009)

12. Reichert, M., Dadam, P.: Enabling Adaptive Processes with ADEPT2. In: Cardoso, J.; van der Aalst, W.M.P. (Eds.): Handbook of Research in Business Process Modeling. Information Science Reference (an imprint of IGI Global), pp. 173-203 (2009)

13. Igler, M. Moura, P. Faerber, M. Zeising, M. Jablonski, S.: Modeling and planning collaboration using organizational constraints. Proc. Collaborative Computing: Networking, Applications and Worksharing (CollaborateCom 2010), Chicago, Ill., Oct. 2010

14. Russell, N., ter Hofstede, A.H.M., van der Aalst, W.M.P., Mulyar, N.: Workflow ControlFlow Patterns: A Revised View. BPM Center Report BPM-06-29, BPMcenter.org, 2006

15. Sadiq, S., Sadiq, W., Orlowska, M.: Pockets of Flexibility in Workflow Specification. In: Proc. of the $20^{\text {th }}$ Int'l Conf. on Conceptional Modeling, LNCS, vol. 2224, pp. 513-526, Springer, Berlin Heidelberg (2001)

16. Hallerbach, A., Bauer, T., Reichert, M.: Configuration and Management of Process Variants. In: vom Brocke, J., Rosemann, M. (eds.): Handbook of Business Process Management (2009)

17. R.S. Mans, N.C. Russell, W.M.P. van der Aalst, A.J. Moleman, and P.J.M. Bakker: Proclets in Healthcare. BPM Center Report BPM-09-05, BPMcenter.org, 2009

18. Reuter, C.: Composition of Semantic Process Fragments to Domain-related Process Families. In: van Bommel, P., Hoppenbrouwers, S., Overbeek, S., Proper, E., Barjis, J. (eds.): The Practice of Enterprise Modeling (PoEM 2010), LNBIP 68, pp. 61-75, Springer Berlin-Heidelberg (2010)

19. Reuter, C.: Modellierung und dynamische Adaption klinischer Pfade auf Basis Semantischer Prozessfragmente (SPF). PhD thesis, Technical University of Dortmund, Germany, Computer Science Faculty, 2011 